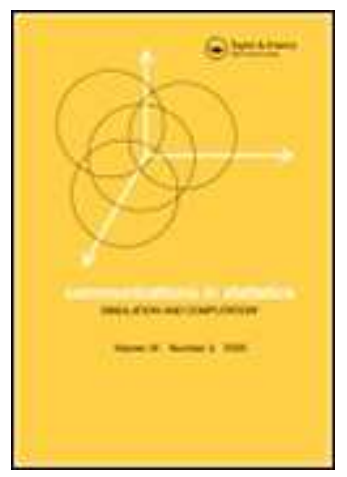

\title{
ON THE RUN LENGTH OF A STATE-SPACE CONTROL CHART FOR MULTIVARIATE AUTOCORRELATED DATA
}

\begin{tabular}{|r|l|}
\hline Journal: & Communications in Statistics - Simulation and Computation \\
\hline Manuscript ID: & LSSP-2008-0309.R2 \\
\hline Manuscript Type: & Review Article \\
\hline Author: & 13 -May-2009 \\
\hline Complete List of Authors: & $\begin{array}{l}\text { VARGAS VARGAS, MANUEL; UNIVERSIDAD DE CASTILLA-LA } \\
\text { MANCHA, Facultad de Ciencias Económicas y Empresariales } \\
\text { ALFARO NAVARRO, JOSE-LUIS; UNIVERSIDAD DE CASTILLA-LA } \\
\text { MANCHA, Facultad de Ciencias Económicas y Empresariales } \\
\text { MONDEJAR JIMENEZ, JOSE; UNIVERSIDAD DE CASTILLA-LA } \\
\text { MANCHA, Facultad de Ciencias Sociales }\end{array}$ \\
\hline Keywords: & $\begin{array}{l}\text { Statistical Process Control, Multivariate Control Chart, State-Space } \\
\text { Model, Average Run Length }\end{array}$ \\
\hline Abstract: & $\begin{array}{l}\text { The literature on statistical process control (SPC) describes the } \\
\text { negative effects of autocorrelation in terms of false alarms. This has } \\
\text { been treated by the individual modelling of each series or the } \\
\text { application of VAR models. In the former case, the analysis of the } \\
\text { cross correlation structure between the variables is altered. In the } \\
\text { latter, the filtering process can modify the weakest relations. In } \\
\text { order to improve these aspects, state-space models have been } \\
\text { introduced in MSPC. This paper presents a proposal for building a } \\
\text { innovations control chart, estimating its average run length to } \\
\text { highlight its advantages over the VAR approach. }\end{array}$ \\
\hline & \\
\hline
\end{tabular}

\section{今 ScholarONE" \\ Manuscript Central}




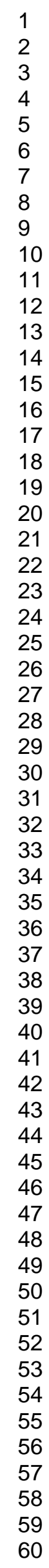




\title{
ON THE RUN LENGTH OF A STATE-SPACE CONTROL CHART FOR MULTIVARIATE AUTOCORRELATED DATA
}

\author{
Manuel Vargas ${ }^{1}$, José Luis Alfaro ${ }^{* 1}$ and José Mondéjar ${ }^{2}$ \\ 1 Facultad de Ciencias Económicas y Empresariales, Universidad de Castilla-La \\ Mancha, Pza. de la Universidad nº 1, 02071, Albacete, España. \\ 2 Facultad de Ciencias Sociales, Universidad de Castilla-La Mancha, Avda. de los \\ Alfares no 44,16002, Cuenca, España.
}

\begin{abstract}
The literature on statistical process control (SPC) describes the negative effects of autocorrelation in terms of the increase in false alarms. This has been treated by the individual modelling of each series or the application of VAR models. In the former case, the analysis of the cross correlation structure between the variables is altered. In the latter, if the cross correlation is not strong, the filtering process may modify the weakest relations. In order to improve these aspects, state-space models have been introduced in MSPC. This paper presents a proposal for building a control chart for innovations, estimating its average run length to highlight its advantages over the VAR approach mentioned above.
\end{abstract}

KEY WORDS: Statistical Process Control, Multivariate Control Chart, StateSpace Model, Average Run Length.

\footnotetext{
${ }^{*}$ Corresponding author.
} 


\section{INTRODUCTION}

The main aims of statistical process control methods is to minimise the frequency of false alarms and quickly detect special sources of variation. Shewhart control charts are designed for the first purpose, but under the condition that the observations analysed are independent. However, in practice this is not a general condition, as there are a wide range of autocorrelated processes. This phenomenon limits its use and has led to the development of statistical models adapted to this phenomenon.

Traditionally, the treatment of autocorrelation focused on the use of empirical control limits or on the filtering of information using univariate time series models (Harris and Ross, 1991; Montgomery and Mastrangelo, 1991; Maragah and Woodall, 1992; Box and Luceño, 1997; Lu and Reynolds, 1999; Jiang, 2001; West and Jarrett, 2004). The earliest multivariate developments for treating autocorrelation consisted of the individual modelling of each series or the application of VAR modelling (Dyer et al., 2003; Jiang, 2004; Kalgonda and Kulkarni, 2004; Noorossana and Vaghefi, 2006; Jarrett and Pan, 2007a, b; Pan and Jarrett, 2007. In the former case, the analysis of the structure of cross correlation between the variables, the great contribution of the multivariate approach, is altered. In the latter case, if the original cross correlation is not strong, the filtering process may modify the weakest relations. Moreover, the autocorrelation may reflect the existence of some "latent factor" or "inertia" in the process. In such a case, analysis of the residuals will reveal the "filtered" behaviour of the process, i.e., the behaviour of the non-foreseeable or innovational part, without going into an analysis of the "inertia factor". 
On a simultaneous basis, the works of Negiz and Cinar (1997), Dorsey and Lee (2003), Lee and Dorsey (2004), Pan and Jarrett (2004), Alfaro (2005), Triantafyllopoulos (2006), Xie et al. (2006) and Zantek et al. (2007) introduced multiple time series state-space modelling in statistical process control. This modelling allows to eliminate the autocorrelation existing in the data, preserving a great amount of information in the residuals, that in this methodology are denoted as innovations, and also allowing to describe the existence of inertia in the process by building a control chart for the states variables, estimate with the state-space model.

This paper will propose state-space treatment for autocorrelated multivariate processes. The second section describes the new control chart for innovations that is more efficient than others existing in the literature. The third section includes an estimation of the average run length (ARL) for this chart, obtained by simulation for various scenarios established according to different levels of correlation and autocorrelation. Finally, the fourth section contains the general conclusions of this paper.

\section{THE MULTIVARIATE STATE-SPACE CONTROL CHART}

The interest in state-space representation began with Kalman's works (1960, 1963), although various approaches were subsequently developed for identifying and estimating these models. As well as using the maximum likelihood method, Akaike $(1975,1976)$ proposed an approach based on canonical correlation, which may lead to non-equivalent representations when the order of the endogenous variables was changed. Later on, Aoki (1987) developed an approach based on the decomposition of the estimated autocovariance matrix in singular values. Algorithms for identifying 
subspaces have also been developed in several papers. For a complete reviewed of these see Vargas (1999), García (2005) and the cited references.

In statistical process control, the basic motivation for using state-space modelling lies in the double reasons for sample autocorrelation. On the one hand, when the sample procedure causes autocorrelation in the data, it is appropriated to apply a filter to eliminate this, preserving the greatest possible amount of information and building a control chart on the residuals. However, if the autocorrelation reflects the existence of a "latent factor" or "inertia" inherent to the process, which provides basic information on it, the residuals of a filtering operation are not statistically optimal, because of a fundamental part of the process is omitted. This case requires an efficient estimation of the "inertia factor", as well as the building of a chart to control it. In this paper, we focus on the control chart for innovations, comparing its ARL to that of charts obtained on the basis of residuals obtained from VAR models.

Given the estimation of a state-space model, the resulting innovations $\left(\varepsilon_{t}\right)$ are comparable to the residuals from VAR modelling. They describe the innovational or non-foreseeable behaviour of the process, controlling the existence of special causes whose effects are of great magnitude and short duration in time. The state-space methodology allows to preserve the multivariate approach along with a more parsimonious representation, an ideal situation in quality control.

In this framework, it is crucial to estimate "correctly" the state variables which are sufficient statistics for the dynamics of the process and they determine the statistical properties of the residuals. To estimate state-space models in statistical process control, a number of approaches exist (Negiz and Cinar, 1997; Dorsey and Lee, 2003; Lee and 
Dorsey, 2004; Pan and Jarrett, 2004; Triantafyllopoulos, 2006; Xie et al., 2006; Jarrett and Pan, 2007a; Zantek et al., 2007), with different algorithms that provide different statistical behaviour.

Therefore, given a p-dimensional autocorrelated process, $Y_{t}$, its state-space representation:

$$
\begin{aligned}
& X_{t+1}=F_{t} X_{t}+G_{t} \varepsilon_{t} \\
& Y_{t}=H_{t} X_{t}+\varepsilon_{t}
\end{aligned}
$$

allows to estimate the innovations in the process, as explained in the appendix. Given the properties of the algorithm used, the innovations $\left(\varepsilon_{\mathrm{t}}\right)$ are asymptotically normal and uncorrelated, with zero mean and variance $\Psi$. In these conditions, we can build a $T^{2}$ Hotelling control chart using the estimation of the matrix $\Psi$ provided by the state-space filtering process, by means of the expression:

$$
T_{\varepsilon_{i}}^{2}=\varepsilon_{i}^{\prime} \hat{\Psi}^{-1} \varepsilon_{i}
$$

When the innovations are uncorrelated, the $\mathrm{T}^{2}$ statistics follows the Beta distribution in the Phase I (and F distribution in the Phase II) and we can get the control limit as:

$$
\operatorname{LSC}\left(T_{\varepsilon_{i}}^{2}\right)=\frac{(m-1)^{2}}{m} \beta_{\alpha, p / 2,(m-p-1) / 2}
$$

But, when we use the estimation $\hat{\psi}$, the innovations are uncorrelated only asymptotically. In this case, the corresponding control limit is:

$$
\operatorname{LSC}\left(T_{\varepsilon_{i}}^{2}\right)=\chi^{2}(p)
$$

which is similar to the one obtained for the original series, as the number of variables is equal to the number of innovations and no observations are lost as a consequence of the filtering. 
This control chart allows us to detect the special causes for variation that present an effect of relative magnitude and short duration in time. This chart is similar to the residuals control chart developed in Jarrett and Pan (2007b) and Pan and Jarrett (2007), although there are some aspects that are different due to the statistical methodology used in estimation. The algorithm for estimating the matrices in the model guarantees the minimality of the state vector, by balancing the grammians of the observability and controllability matrices, lending these characteristics to the estimated system (guaranteeing minimality). Also, the use of the Kalman filter ensures that the states constitute the optimal linear estimation (in the minimum quadratic sense of the expression) of the process dynamic. This all comes together in obtaining innovations that contain all of the complementary information, thereby allowing for thorough analysis.

As show Xie et al. (2006), the use of ARMA filters ensures the efficient elimination of autocorrelations, but the cross correlations still exist and the residuals are not independent. In contrast to this filter, the state-space model is enough to remove the auto and cross correlation. With the algorithm proposed, the statistical properties of the state variables ensures the "minimum" correlation in the residuals.

To compare the statistical properties of the control charts, it is usual to examine their performance in terms of the ARL (Wardell et al., 1994; Javaheri and Houshmand, 2001; Jiang, 2001; Loredo et al., 2002; Shu et al., 2004; Snoussi et al., 2005; Tsung et al., 2006; Snoussi and Limam, 2007). Based on the ARL as criteria of control charts performance, we compare the proposed alternative control chart with the obtained by means of VAR modelling. We analyse the possible effect of cross and autocorrelation through the simulation under different levels of both. 


\section{SIMULATION STUDY}

The simulation process has been developed using R-Program. More specifically, the algorithm used for simulated a multivariate normal process are developed in the function rmvnorm in the mvtnorm package written by Alan Genz in $R$ software. For adjusted and estimated a VAR model by least square, we have used the function estVARXls in the dse package written in $R$ by Paul Gilbert. All these packages are available from the $R$ project website (http://rprojects.org/). The state-space model estimation, developed in the appendix, is also implemented in $\mathrm{R}$ with a function developed by the authors (available upon request)

To simplify, without losing generality, we opted to simulate three-dimension processes with the same level of correlation between the variables. We generated, under a normal multivariate distribution of parameters $\mu_{0}$ and $\Sigma_{0}, 150$ in-control observations. Then, we generated five out-of-control observations $\left(\mu=\mu_{l} \neq \mu_{0}\right)$, with changes in the mean vector of $0,1,2$ and 3 standard deviations, changes usually considered in statistical process control. Finally, we generated another sequence of 5000 in-control observations to determine the run length.

To analyse the detection capability of the innovations control chart we have considered different scenarios by varying the levels of cross correlation and autocorrelation. In the first case, we focused on positive correlation, given it's the most usual in the production processes. Therefore, correlations of $0,0.3,0.5,0.7$ and 0.9 were considered, in order to detect if the level of correlation affects the detection capacity of control charts. These information are introduced in the Hotelling's $\mathrm{T}^{2}$ statistic via the covariance matrix. 
Also, we analyse four possibilities of the level of autocorrelation, corresponding to coefficients of $0.3,0.5,0.7$ and 0.9 , given its greater impact on the properties of control charts in terms of false alarms. For each combination, the simulation was repeated 1000 times, which allows to obtain the ARL for the control charts exposed.

Once the series had been simulated, we have adjusted and estimated two models: a VAR model by least square by means of the estVARXls function in the $d s e$ package in $R$, following the methodology used in Jarrett and Pan (2007b) and Pan and Jarrett (2007); and a state-space model using the proposed methodology, implemented in R. The control limits were estimated to attain a value of 280 for the in-control average run length $\left(\mathrm{ARL}_{0}\right)$ and the run length was calculated for each series. The averages in each case are shown in tables 1 to 4, which depict the improvement, in terms of ARL, in the control chart proposed compared to the traditional one based on the VAR methodology.

\section{INSERT TABLE 1}

The table 1 shows that if the process is in-control, both the VAR and the statespace approaches present similar behaviour, with non-significant differences in $\mathrm{ARL}_{0}$. In this case, the greatest differences appear with high levels of correlation and autocorrelation and that the behaviour of the innovations control chart proposed is better.

\section{INSERT TABLE 2}

The table 2 shows that when the change in the means are smaller, the differences between the two methods are not statistically significant. The ARL takes values around 140 in the both methods. 
The tables 3 and 4 show that when changes occur in the mean of the process, the state-space chart improves its behaviour, with an increase in relative efficiency as the magnitude of the change increases. Obviously, if we express these changes by means of the non-centrality parameter, affected by the existence of cross correlation between the variables, then we can not directly compare the values for the ARL. This fact manifests itself in tables 2, 3 and 4, where the run length of the models increases as the cross correlation increases, with the consequent reduction in the non-centrality parameter.

\section{INSERT TABLES 3 and 4}

Simulation results show that the state-space model formulation provides better ARL behaviour than residual charts based on VAR methodology (see figure 1). In general, the $\mathrm{ARL}_{0}$ is similar for the two control charts, as they originate from the innovations in two equivalent methodologies for modelling statistical processes. When a change occurs in the mean of the process, the behaviour of the control charts starts to diverge, whereas the ARL of the chart proposed is lower than the one that is built under the VAR approach. This difference is further accentuated as the non-centrality parameter increases, which is dependent on the distance between the real mean and the in-control mean.

Although the existence of autocorrelation alters the ARL of both charts, the results obtained with the methodology proposed are more homogeneous and stable, reflecting greater resistance to this problem. On the other hand, the existence of cross correlation presents no other effect than the reduction in the non-centrality parameter, with both charts equally affected.

\section{INSERT FIGURE 1}




\section{SUMMARY}

The traditional proposals to eliminate the negative effect of autocorrelation in statistical quality control present problems related to the loss of the multivariate approach, the parsimony of adjusted models and the poor behaviour in the presence of positive autocorrelation. State-space modelling constitutes an alternative that allows to maintain the multivariate perspective, requiring the estimation of less parameters and with better behaviour in terms of ARL.

We developed a simulation to analyse the behaviour of the control chart proposed in the event of changes in the mean of the process, enabling comparison with the VAR control chart and the study of the effect of the non-independence between the observations.

The results of this indicate that the behaviour of the state-space innovations control chart is more adequate than the residuals control chart obtained by means of VAR modelling. To be specific, the innovations control chart presents better behaviour in terms of out-of-control ARL and greater resistance to the existence of positive autocorrelation. These advantages are accentuated the greater the importance of the change in the mean of the process, situations in which the methodology proposed in this paper is a significant improvement on the traditional VAR modelling.

This paper is a first approach to the behaviour of control chart based on innovations of a state-space model. As such, it has not approached the effect of changes on the correlation structure of the variables or on their variability. Similarly, future researches should analyse the sensitivity of the results of the simulation to changes in 
the magnitude of the correlation between variables or combinations of different levels of autocorrelation.

\section{REFERENCES}

Akaike, H. (1975). Markovian Representation of Stochastic Processes by Canonical Variables. SIAM Journal on Control and Optimization 13(1):162-173.

Akaike, H. (1976). Canonical Correlation Analysis of Time Series and the Use of an Information Criterion. New York: Academic Press.

Alfaro, J. L. (2005). Control estadístico de la calidad en procesos multivariantes autocorrelacionados. Una aplicación en la industria cuchillera de Albacete. Universidad de Castilla-La Mancha.

Aoki, M. (1987). State-space modelling of time series. New York: Springer-Verlag.

Bartlett, M. S. (1939). A note on tests of significance in multivariate analysis. Proceeding of the Cambridge Philosophical Society 35: 180 - 185.

Bauer, D. and Wagner, M. (2002). Estimating cointegrated systems using subspace algorithms. Journal of Econometrics 111: 47-84.

Box, G. E. P. and Luceño, A. (1997). Statistical Control by Monitoring and Feedback Adjustement. New York: Wiley.

Dorsey, A. W. and Lee, J. H.(2003). Building Inferential Prediction Models of Batch Processes Using Subspace Identification. Journal of Process Control 13(5): 397406. 
Dyer, J. N., Connerly, M. D. and Adams, B. M. (2003). A simulation study and evaluation of multivariate forecast based control charts applied to ARMA processes. Journal of Statistical Computation and Simulation 73: 709-724.

García, A. (2005). Identificación de Modelos para Series Temporales mediante Métodos de Subespacios. Tesis Doctoral. Universidad Complutense de Madrid. Available in: http://www.ucm.es/BUCM/tesis/cee/ucm-t28292.pdf

Harris, T. J. and Ross, W. H. (1991). Statistical Process Control Procedures for Correlated Observations. Canadian Journal of Chemical Engineering 69: 48-57.

Jarrett, J. and Pan, X. (2007a). Monitoring Variability and Analyzing Multivariate Autocorrelated Processes. Journal of Applied Statistics 34(4): 459-469.

Jarrett, J. and Pan, X. (2007b). The quality control chart for monitoring multivariate autocorrelated processes. Computational Statistics \& Data Analysis 51(8): 38623870.

Jiang, W. (2001). Average Run Length Computation of ARMA Charts for Stationary Processes. Communications in Statistics. Simulation and computation 30(3): 699716.

Jiang, W. (2004). Multivariate control charts for monitoring autocorrelated processes. Journal of Quality Technology 36: 367-379.

Kalgonda, A. A. and Kulkarni, S. R. (2004). Multivariate quality control chart for autocorrelated processes. Journal of Applied Statistics 31: 317-327.

Kalman, R. E. (1960). A New Approach to Linear Filtering and Prediction Problems. Journal of Basic Engineering. Transaction of ASME series D 82: 35-45. 
Kalman, R. E. (1963). New Methods in Weiner Filtering Theory. In: Bogdanoff, J. L. and Kozin, F. (Eds), Proceedings of the First Symposium of Engineering Applications of Random Function Theory and Probability. New York: Wiley.

Lee, J. H. and Dorsey, A. W. (2004). Monitoring of batch processes through state-space models. AIChE Journal 50(6): 1198-1210.

Loredo, E. N., Jaerkpaporn, D. and Borror, C. M. (2002). Model-based control chart for autoregressive and correlated data. Quality and Reliability Engeenering International 18(6): 489-496.

Lu, C. W. and Reynolds, M. R. (1999). Control Charts for Monitoring the Mean and Variance of Autocorrelated Processes. Journal of Quality Technology 31: 259-274.

Maragah, H. O. and Woodall, W. H. (1992). The effect of Autocorrelation on the Retrospective X-Chart. Journal of Statistical Computation and Simulation 40(1): $29-42$.

Mondéjar, J. and Vargas, M. (2006). Análisis de tendencias comunes y cointegración en espacio de estados. Contribuciones a la Economía september. Available in: http://www.eumed.net/ce/

Montgomery, D. C. and Mastrangelo, C. M. (1991). Some Statistical Process Control Methods for Autocorrelated Data. Journal of Quality Technology 23: 179-204.

Negiz, A. and Cinar, A. (1997). Statistical monitoring of multivariate dynamic processes with state-space models. AIChE Journal 43(8): 2002-2020.

Noorossana, R. and Vaghefi, S. J. M. (2006). Effect of autocorrelation on performance of the MCUSUM control chart. Quality and Reliability Engineering International 2 (2):191-197. 
Pan, X. and Jarrett, J. (2004). Applying State Space into SPC: Monitoring Multivariate Time Series. Journal of Applied Statistics 31(4): 397-418.

Pan, X. and Jarrett, J. (2007). Using vector autoregressive residuals to monitor multivariate processes in the presence of serial correlation. International Journal of Production Economics 106(1): 204-216.

Shu, L., Tsung, F., and Tsui, K. L. (2004). Run-Length Performance of Regression Control Charts with Estimated Parameters. Journal of Quality Technology 36(3): 280-292.

Snoussi, A., Ghourabi, M. E. and Limam, M. (2005). On SPC for short run autocorrelated data. Communications in Statistics: Simulation and Computation 34(1): 219-234.

Snoussi, A. and Limam, M. (2007). The Change Point Model: SPC Method for Short Run Autocorrelated Data. Quality Technology \& Quantitative Management 4(3): 313-329.

Triantafyllopoulos, K. (2006). Multivariate Control Charts Based on Bayesian State Space Models. Quality and Reliability Engineering International 22: 693-707.

Tsung, F., Zhao, Y., Xiang, L., and Jiang, W. (2006). Improved Design of Proportional Integral Derivative Charts. Journal of Quality Technology 38(1): 31-44.

Vargas, M. (1999). Modelización de series temporales múltiples en espacio de estados. Análisis de procesos no estacionarios y cointegración. Universidad de Castilla-La Mancha.

Wardell, D. G., Moskowitz, H. and Plante, R. D. (1994). Run-length distribution of special-cause control charts for correlated processes. Technometrics 36:1084-1105. 
West, D. and Jarrett, J. (2004). The Impact of First Order Positive Autoregression on Process Control. International Journal of Business and Economics 3(1): 29-37.

Xie, L., Zhang, J. and Wang, S. (2006). Investigation of Dynamic Multivariate Chemical Process Monitoring. Chinese Journal of Chemical Engeenering 14(5): $559-568$.

Zantek, P., Li, S. and Chen, Y. (2007). Detecting multiple special causes from multivariate data with applications to fault detection in manufacturing. IIE Transactions 39(8): 771-782. 


\section{Tables}

Table 1. In-control average run length $\left(A R L_{0}\right)$

\begin{tabular}{|c|c|c|c|c|c|c|}
\hline & & & \multicolumn{4}{|c|}{ Auto-correlation level } \\
\hline & & & 0.3 & 0.5 & 0.7 & 0.9 \\
\hline \multirow{10}{*}{ 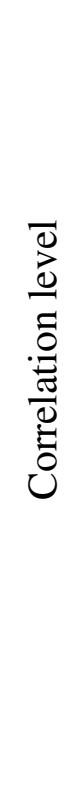 } & \multirow{2}{*}{0} & VAR & 267.774 & 274.172 & 277.545 & 286.145 \\
\hline & & St-Sp & 262.543 & 278.252 & 289.146 & 291.676 \\
\hline & \multirow{2}{*}{0.3} & VAR & 271.775 & 267.401 & 274.946 & 279.617 \\
\hline & & $\mathrm{St}-\mathrm{Sp}$ & 277.540 & 262.502 & 283.131 & 271.794 \\
\hline & \multirow{2}{*}{0.5} & VAR & 278.312 & 275.072 & 274.208 & 265.448 \\
\hline & & $\mathrm{St}-\mathrm{Sp}$ & 279.877 & 283.119 & 278.749 & 280.215 \\
\hline & \multirow{2}{*}{0.7} & VAR & 264.815 & 280.609 & 267.846 & 270.358 \\
\hline & & $\mathrm{St}-\mathrm{Sp}$ & 265.034 & 285.444 & 280.347 & 283.548 \\
\hline & \multirow{2}{*}{0.9} & VAR & 280.033 & 279.645 & 285.984 & 267.749 \\
\hline & & $\mathrm{St}-\mathrm{Sp}$ & 292.700 & 283.018 & 286.223 & 275.478 \\
\hline
\end{tabular}

Table 2. ARL with changes in the mean vector of one standard deviations

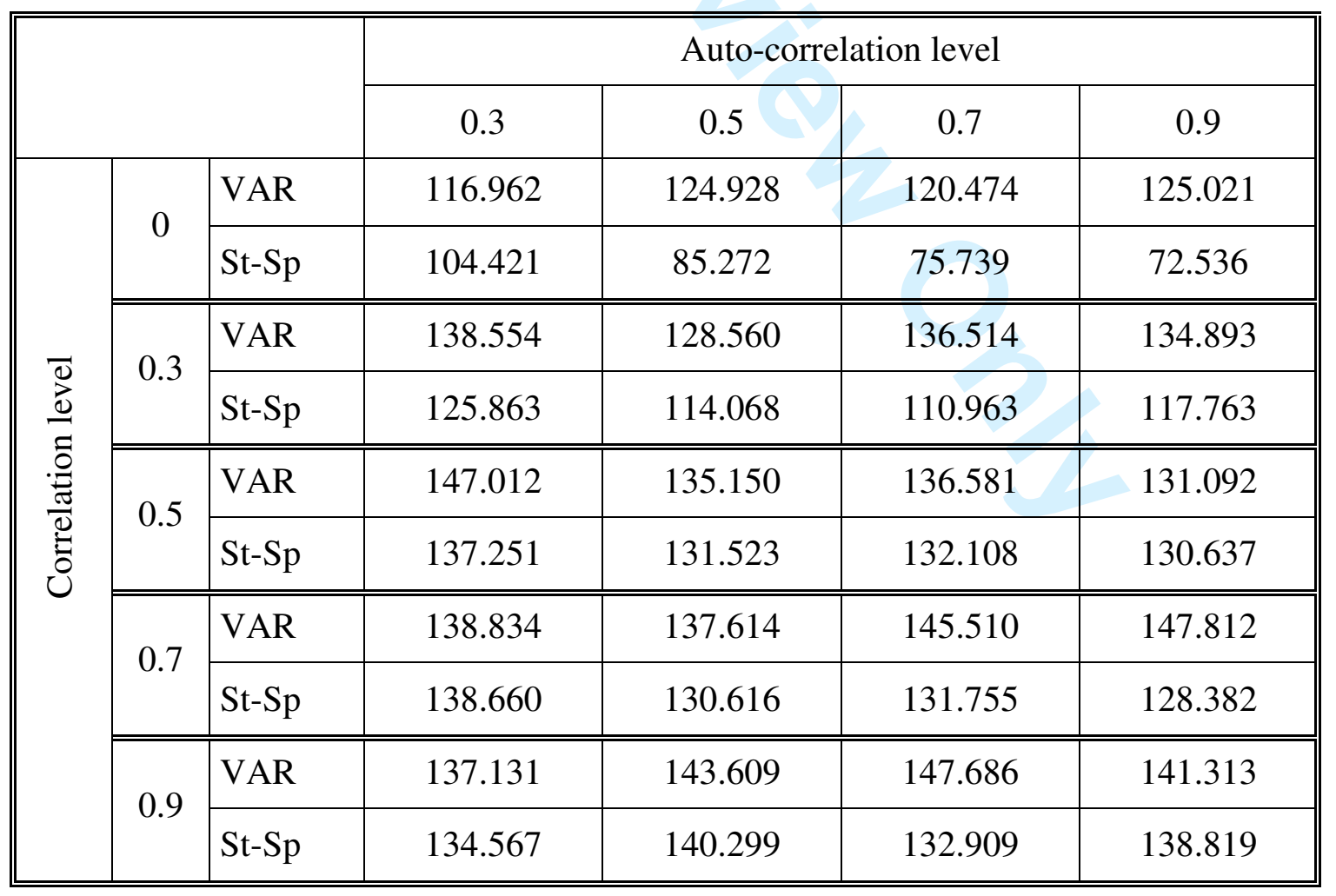


Table 3. ARL with changes in the mean vector of two standard deviations

\begin{tabular}{|c|c|c|c|c|c|c|}
\hline & \multicolumn{4}{|c|}{ Auto-correlation level } \\
\hline & & & 0.3 & 0.5 & 0.7 & 0.9 \\
\hline \multirow{10}{*}{ 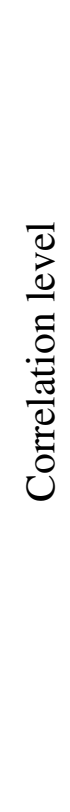 } & \multirow{2}{*}{0} & VAR & 28.425 & 24.549 & 29.251 & 25.981 \\
\hline & & $\mathrm{St}-\mathrm{Sp}$ & 5.509 & 2.739 & 1.782 & 2.116 \\
\hline & \multirow{2}{*}{0.3} & VAR & 64.202 & 66.294 & 65.677 & 65.091 \\
\hline & & St-Sp & 29.183 & 14.305 & 27.284 & 26.932 \\
\hline & \multirow{2}{*}{0.5} & VAR & 80.772 & 83.386 & 84.757 & 82.849 \\
\hline & & $\mathrm{St}-\mathrm{Sp}$ & 45.516 & 49.375 & 47.983 & 48.514 \\
\hline & \multirow{2}{*}{0.7} & VAR & 96.023 & 97.646 & 93.438 & 92.584 \\
\hline & & $\mathrm{St}-\mathrm{Sp}$ & 68.132 & 64.029 & 68.272 & 64.416 \\
\hline & \multirow{2}{*}{0.9} & VAR & 103.403 & 104.682 & 105.035 & 105.314 \\
\hline & & $\mathrm{St}-\mathrm{Sp}$ & 72.352 & 76.127 & 72.733 & 74.181 \\
\hline
\end{tabular}

Table 4. ARL with changes in the mean vector of three standard deviations

\begin{tabular}{|c|c|c|c|c|c|c|}
\hline & \multicolumn{4}{|c|}{ Auto-correlation level } \\
\hline & & & 0.3 & 0.5 & 0.7 & 0.9 \\
\hline \multirow{10}{*}{ 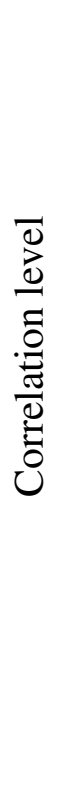 } & \multirow{2}{*}{0} & VAR & 1.779 & 2.622 & 1.603 & 1.316 \\
\hline & & St-Sp & 1.049 & 1.070 & 1.116 & 1.200 \\
\hline & \multirow{2}{*}{0.3} & VAR & 27.709 & 35.329 & 42.113 & 29.287 \\
\hline & & $\mathrm{St}-\mathrm{Sp}$ & 1.469 & 1.423 & 2.115 & 2.002 \\
\hline & \multirow{2}{*}{0.5} & VAR & 71.417 & 71.214 & 91.722 & 73.198 \\
\hline & & $\mathrm{St}-\mathrm{Sp}$ & 5.327 & 3.880 & 8.015 & 5.983 \\
\hline & \multirow{2}{*}{0.7} & VAR & 122.107 & 126.480 & 98.197 & 101.212 \\
\hline & & St-Sp & 25.137 & 21.126 & 23.130 & 25.506 \\
\hline & \multirow{2}{*}{0.9} & VAR & 144.310 & 148.512 & 168.222 & 139.027 \\
\hline & & St-Sp & 39.168 & 43.686 & 49.456 & 41.334 \\
\hline
\end{tabular}




\section{FIGURE}

Figure 1. ARL for VAR and State-Space residuals control charts

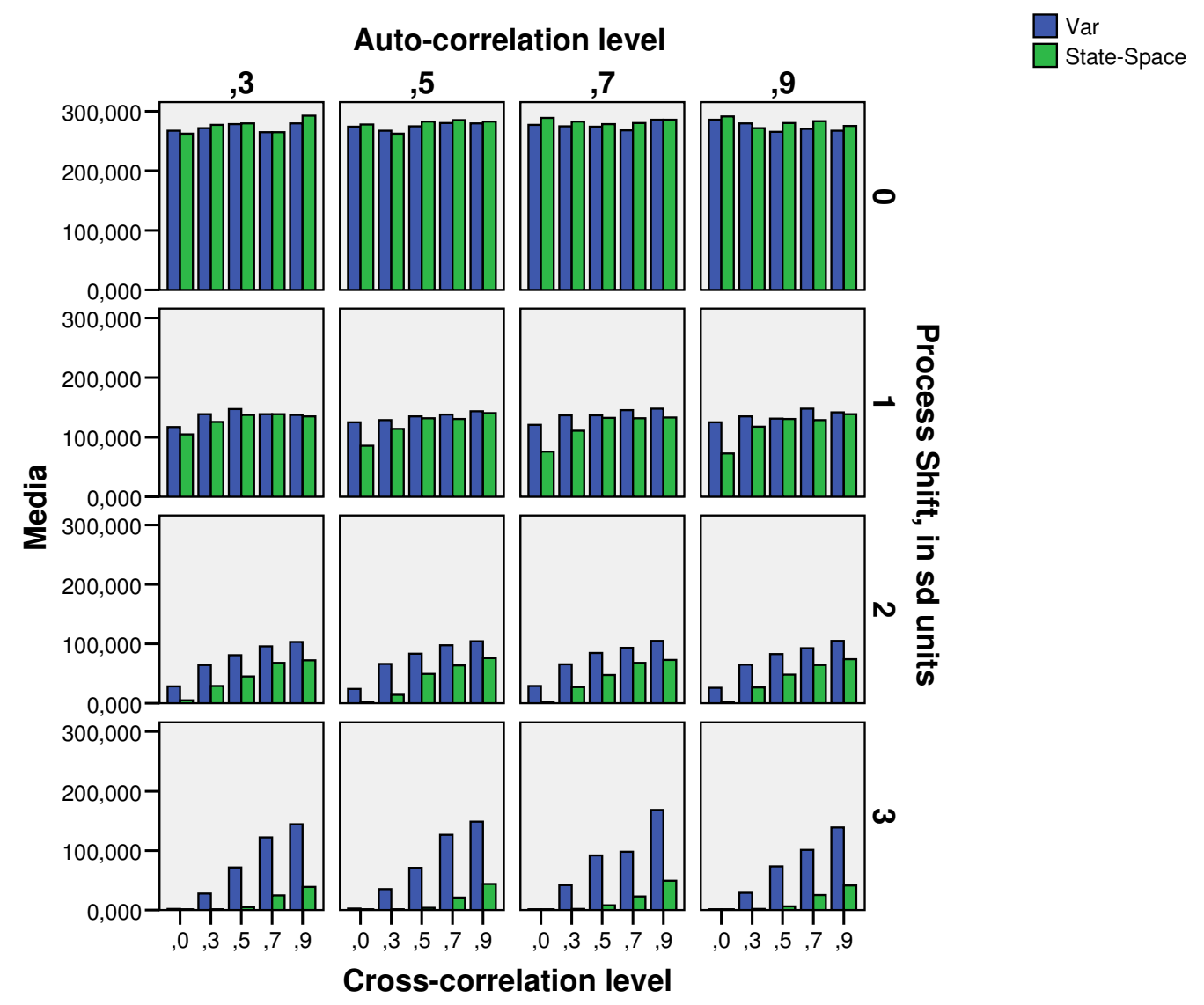




\section{APPENDIX I: A Brief on Identification of State-Space Models}

A discrete linear system in state-space may be defined by means of an innovational model:

$$
\begin{aligned}
& X_{t+1}=F_{t} X_{t}+G_{t} \varepsilon_{t} \\
& Y_{t}=H_{t} X_{t}+\varepsilon_{t}
\end{aligned}
$$

where $H_{t}, F_{t}, G_{t}$ are the real matrices of dimensions (pxn), (nxn) and (nxp) respectively, $\mathrm{p}$ is the number of variables, $\mathrm{n}$ the number of observations and we assume, for simplicity, that $E\left[Y_{t}\right]=0$. In this model, $\varepsilon_{t}$ is the innovational process of $Y_{t}$ that does not present autocorrelation and $\mathrm{X}_{\mathrm{t}}$ is the state vector, a sufficient statistic for the dynamics of the system.

The specification for this model consists of three stages: determining the dimension of the system, estimating the matrices and finally, estimating the state vector. In statistical quality control, determining the dimension of the system is fundamental and therefore different algorithms based on canonical correlation coefficients or information criteria have been used. In this paper, we opted to use the block Hankel matrix, which in practice is usually composed of sample estimations of autocovariances $\Gamma_{\ell}=E\left[Y_{t+\ell} Y^{\prime}\right]$ for $\ell=0,1,2, \ldots$

$$
\hat{H}_{N_{f}}^{N_{p}}=E\left[Y_{t, N_{f}}^{+} Y_{t-1, N_{p}}^{-}\right]=\left(\begin{array}{cccc}
\Gamma_{1} & \Gamma_{2} & \cdots & \Gamma_{N_{p}} \\
\Gamma_{2} & \Gamma_{3} & \cdots & \Gamma_{N_{p}+1} \\
\vdots & \vdots & \vdots & \cdots \\
\Gamma_{N_{f}} & \Gamma_{N_{f}+1} & \cdots & \Gamma_{N_{p}+N_{f}-1}
\end{array}\right)
$$

of dimension $\left(\mathrm{pN}_{\mathrm{f}} \times \mathrm{pN} \mathrm{p}_{\mathrm{p}}\right.$ ), and prefixed values $N_{p}$ and $N_{f}$, and

$$
\begin{aligned}
& Y_{t, N_{f}}^{+}=\left(Y_{t}^{\prime}, Y_{t+1}^{\prime}, \ldots, Y_{t+N_{f}-1}^{\prime}\right)^{\prime} \\
& Y_{t-1, N_{p}}^{-}=\left(Y_{t-1}^{\prime}, Y_{t-2}^{\prime}, \ldots, Y_{t-N_{p}}^{\prime}\right)^{\prime}
\end{aligned}
$$


By estimating the above matrix by means of the sample auto-correlation matrices $\hat{\Gamma}=n^{-1} \sum_{I=1}^{n} Y_{i+1} Y_{i}^{\prime \prime}$, it is possible to decompose the matrix $\hat{H}_{N_{f}}^{N_{p}}=\hat{U} \hat{\Sigma} \hat{V}^{\prime}$ and use as the estimation for its range the number of significantly positive singular values in the matrix $\hat{\Sigma}$, which will coincide with the dimension of the state vector. In this regard, it is possible to use an approximation of the statistical distribution of the canonical correlation coefficients proposed in Bartlett (1939) by means of the statistic:

$$
B_{\hat{n}}=-\left\{T-\frac{1}{2}\left[p\left(N_{p}+N_{f}\right)+1\right]\right\} \ln \prod_{j>\hat{n}+1}\left(1-\gamma_{j}^{2}\right) \rightarrow \chi_{\left(p N_{f}-\hat{n}\right)\left(p N_{p}-\hat{n}\right)}^{2}
$$

where $\gamma_{\mathrm{j}}$ are the canonical correlation coefficients.

On the basis of this statistic, a sequential contrast is established for $\hat{n}=1,2, \ldots$ until the $\mathrm{H}_{0}$ is not refused, which establishes that there are only $n$ strictly positive coefficients, in a value $\hat{n}$ that is considered to be the dimension of the state-space model.

In order to obtain an estimation of the matrices in the system, Bauer and Wagner (2002) propose another approach based on the prior estimation of a regression equation. This algorithm presents the disadvantage that it tends to reduce the variability in series and may alter the determination of points out of control. This situation led us to use a modified version of the algorithm started in Aoki (1987), which may be consulted in its complete form in Vargas (1999) or Mondéjar and Vargas (2006).

As the states are not observable, they may be rotated without changing the algebraic validity of the model. To identify the coefficients by making the observability - controllability factorisation equal to the decomposition of the Hankel matrix in singular values means to implicitly select a system of coordinates for the state. This selection is called a balanced representation and it constitutes a major difference 
between the developed algorithm and other procedures. This property guarantees that a size that is smaller than the correct size is specified for the state vector, the estimations for $F, H$ and $\Omega$ would be an approximation of a lower order than the real matrices of the system. In this case, this lower dimension model includes the most important effects and estimates them on a consistent basis. On the other hand, if the dimension of the estimated model is higher than the correct dimension, some of the system matrices are redundant. The rest remain valid and thus it is not necessary to recalculate them.

Once the order and the matrices of the system have been determined, the Kalman filter is used to generate the series of values for the state vector. This is a fundamental element in statistical quality control as it allows to obtain the value of innovations on a recursive and efficient basis. 